\title{
SOME INVERSE SPECTRAL RESULTS FOR SEMI-CLASSICAL SCHRÖDINGER OPERATORS
}

\author{
Victor Guillemin and Alejandro Uribe
}

\begin{abstract}
We show that the Birkhoff normal form of a classical Hamiltonian $H(x, \xi)=$ $\|\xi\|^{2}+V(x)$ at a non-degenerate minimum $x_{0}$ of the potential determines the Taylor series of the potential at $x_{0}$, provided the eigenvalues of the Hessian are linearly independent over $\mathbb{Q}$ and $V$ satisfies a symmetry condition near $x_{0}$. As a consequence, if $x_{0}$ is the unique global minimum of $V$, the low-lying eigenvalues of the semi-classical Schrödinger operator, $-\hbar^{2} \Delta+V(x)$, determine the Taylor series of the potential at $x_{0}$.
\end{abstract}

\section{Introduction}

In this note we will report on some inverse spectral results for the semi-classical Schrödinger operator,

$$
P=P(\hbar)=-\hbar^{2} \Delta+V(x) .
$$

The potential, $V$, in (1.1) will be assumed to be in $C^{\infty}\left(\mathbb{R}^{n}\right)$ and have a unique nondegenerate global minimum, $V(0)=0$, at $x=0$. We will also assume that, for $\epsilon>0$ sufficiently small, $V^{-1}([0, \epsilon])$ is compact. Then, there exists $\hbar_{0}>0$ such that for $\hbar<\hbar_{0}$ the spectrum of $P$ in a small interval, $[0, \delta]$, consists of a finite number of discrete eigenvalues, $E(\hbar)$. In fact, by Weyl's law

$$
\sharp\{E(\hbar) ; 0 \leq E(\hbar) \leq \delta\}=(2 \pi \hbar)^{-n}\left(\operatorname{Vol}\left\{0 \leq\|\xi\|^{2}+V(x) \leq \delta\right\}+o(1)\right)
$$

(see for instance [2], chapter 9).

To what extent do the eigenvalues, (1.2), determine $V$ ? A consequence of the main result of this paper is the following:

Theorem 1.1. Assume $V$ is symmetric with respect to reflections about the coordinate axes, i.e. for any choice of signs

$$
V\left(x_{1}, \ldots, x_{n}\right)=V\left( \pm x_{1}, \ldots, \pm x_{n}\right) .
$$

In addition, assume that

$$
V(x)=\sum_{i=1}^{n} u_{i}^{2} x_{i}^{2}+O\left(|x|^{4}\right),
$$

where the positive numbers $u_{1}, \ldots, u_{n}$ are linearly independent over the rationals. Then the eigenvalues (1.2) with $0<\hbar<\hbar_{0}$ determine the Taylor series of $V$ at $x=0$. In particular, if $V$ is real analytic, these eigenvalues determine $V$.

Received by the editors September 13, 2005.

V.G. supported in part by NSF grant DMS-0408993.

A.U. supported in part by NSF grant DMS-0401064. 
In one degree of freedom this theorem is fairly elementary and has apparently been known for some time. Indeed by Weyl's law, if $n=1$ the eigenvalues, (1.2) with $0<\hbar<\hbar_{0}$, determine the action function

$$
A(E)=\text { Area }\left\{(x, \xi) ; \xi^{2}+V(x) \leq E\right\}
$$

for $E$ in a neighborhood of zero. But

$$
A(E)=\int_{-x(E)}^{x(E)} \sqrt{E-V(x)} d x
$$

where $x(E)$ is the positive solution of the equation $V(x)=E$. It is known (see [1], $\S 12$ ) that the function $A(E)$ determines $V$, and the asymptotics of $A(E)$ as $E \rightarrow 0$ determines the Taylor series of $V$ at the origin, if $V$ is even. ${ }^{1}$

To prove theorem 1.1, we begin by quoting [3]:

Theorem 1.2. ([3]) Let $\rho \in C_{0}^{\infty}(\mathbb{R})$ be a cut-off function which is 1 for $|s|<1 / 2$ and 0 for $|s|>1$. Then the $\hbar \rightarrow 0$ asymptotic expansion of the trace of the operator

$$
\rho\left(\epsilon^{-1} P(\hbar)\right) \exp \left[i t \hbar^{-1} P(\hbar)\right]
$$

for $\epsilon$ and $t$ close to zero, determines the Birkhoff canonical form at $(x, \xi)=(0,0)$ of the classical Hamiltonian $H(x, \xi)=\sum_{i=1}^{n} \xi_{i}^{2}+V(x)$.

Therefore, one is reduced to proving the following (which is the main contribution of this paper):

Theorem 1.3. Modulo the assumption on $V$ above, the Birkhoff canonical form of the classical Hamiltonian

$$
H(x, \xi)=\sum_{i=1}^{n} \xi_{i}^{2}+V(x)
$$

at $(x, \xi)=(0,0)$ determines the Taylor series of $V$ at $x=0$.

To summarize, the asymptotics as $\hbar \rightarrow 0$ of the trace of the operator (1.3) determine the Taylor series of $V$.

The trace of (1.3) is the wave trace localized to an energy interval of size $O(1)$ with respect to $\hbar$. However, the asymptotics of (1.3), for $t$ in an interval of the form $(0, \tau)$ that does not contain periods of periodic trajectories near $(0,0)$ (other than the critical point itself) can be computed entirely in terms of the quantum Birkhoff canonical form of $P$. This is the starting point for the proof of theorem 1.2.

In [4] Sjöstrand shows that, modulo error terms of order $O\left(\hbar^{\infty}\right)$, the eigenvalues of $P(\hbar)$ within $O\left(\hbar^{\delta}\right)$ of the critical value $(\delta>0$ fixed) coincide with the eigenvalues of its quantum Birkhoff canonical form at $(x, \xi)=(0,0)$. We are grateful to one of the referees of this paper for pointing out that Sjostrand's approach to the direct eigenvalue problem for semi-excited states can be reversed to obtain the following alternative version of theorems 1.1 and 1.3:

\footnotetext{
${ }^{1}$ We thank S. Zelditch, P. Etingof and R. de la Llave for bringing these facts to our attention.
} 
Theorem 1.4. With $V$ as in theorem 1.1, given an integer $N>0$ there exists a $\nu>0$ such that the eigenvalues

$$
\left\{E_{j}(\hbar) ; 0 \leq E_{j}(\hbar) \leq \hbar^{\nu}\right\},
$$

for $0<\hbar<\hbar_{0}$, determine the first $N$ terms in the Taylor series of $V$ at zero.

As regards theorem 1.2, we also prove in [3] a version of this theorem involving resonances, and from it one obtains a version of theorem 1.3 which asserts that, in the presence of resonances, one can still recapture from the data (1.2) all the terms in the Taylor series expansion of the potential, $V$, up to the terms where the resonances first occur.

The organization of the paper is as follows. We will review the proof of the existence of the quantum Birkhoff canonical form for $P(\hbar)$ in $\S 2$ and, by carefully examining the $N$-th stage of this proof, show that the first $N$ terms in the classical Birkhoff canonical form determine the $N$-th term in the Taylor series of $V$ at $x=0$.

The last section of this paper $(\S 3)$ is a brief description of a generalization of theorem 1.3 to Riemannian manifolds.

\section{Microlocal Birkhoff canonical forms}

2.1. If $V$ is a potential with the properties described in Theorem 1.1, then microlocally in a neighborhood of $(x, \xi)=(0,0)$ the Schrödinger operator (1.1) can be conjugated by a unitary FIO to a rather simple "quantum Birkhoff normal form". More explicitly, there exist neighborhoods, $\mathcal{O}_{1}$ and $\mathcal{O}_{2}$, of $(x, \xi)=(0,0)$ in $\mathbb{R}^{2 n}$, a canonical transformation

$$
\kappa: \mathcal{O}_{1} \rightarrow \mathcal{O}_{2}, \quad \kappa(0,0)=(0,0)
$$

and a quantization of $\kappa$ by a unitary Fourier integral operator, $\mathcal{U}$, such that microlocallly on $\mathcal{O}_{2}$

$$
\mathcal{U} P \mathcal{U}^{-1}=p\left(\hbar^{2} D_{1}^{2}+x_{1}^{2}, \ldots, \hbar^{2} D_{n}^{2}+x_{n}^{2}, \hbar\right)+Q_{\infty}(\hbar)+R
$$

where:

(1) The smooth function $p$ is an $\hbar$-admissible symbol admitting an asymptotic expansion

$$
p\left(s_{1}, \ldots, s_{n}, h\right) \sim \sum_{j=0}^{\infty} \hbar^{j} p_{j}\left(s_{1}, \ldots s_{n}\right)
$$

where the $p_{j}$ are smooth functions of $n$ variables, and the operator $p\left(\hbar^{2} D_{1}^{2}+\right.$ $\left.x_{1}^{2}, \ldots \hbar^{2} D_{n}^{2}+x_{n}^{2}, \hbar\right)$ is the Weyl quantization of $p\left(\xi_{1}^{2}+x_{1}^{2}, \ldots, \xi_{n}^{2}+x_{n}^{2}\right)$.

(2) $p_{0}$ is of the form:

$$
p_{0}(w)=\sum_{k} u_{k}^{2} w_{k}+\cdots
$$

the dots indicating quadratic and higher order terms.

(3) $Q_{\infty}$ is of order $\infty$ in $\hbar$,

(4) The symbol of $R$ vanishes to infinite order at $(x, \xi)=(0,0)$.

The proof of this theorem can be found in [4]. However, we will go over the details of the proof since they will also be involved in the proof of theorem 1.3. 
2.2. Classical Birkhoff canonical forms. We begin by recalling the construction of the classical Birkhoff canonical form. Conjugating the Hamiltonian (1.4) by the linear symplectomorphism

$$
x_{i} \mapsto u_{i}^{1 / 2} x_{i}, \quad \xi_{i} \mapsto u_{i}^{-1 / 2} \xi_{i}, \quad i=1, \ldots n
$$

one can assume without loss of generality that

$$
H=\sum_{i} u_{i}\left(x_{i}^{2}+\xi_{i}^{2}\right)+V\left(x_{1}^{2}, \ldots, x_{n}^{2}\right)
$$

where $V\left(s_{1}, \ldots, s_{n}\right)=O\left(s^{2}\right)$.

We will prove inductively that for $N=1,2, \ldots$ there exists a neighborhood, $\mathcal{O}$, of $x=0=\xi$, and a canonical transformation, $\kappa: \mathcal{O} \hookrightarrow \mathbb{R}^{2 n}, \kappa(0,0)=(0,0)$, such that

$$
\kappa^{*} H=\sum_{i=1}^{N} H_{i}+R_{N+1}+R_{N+1}^{\prime},
$$

where:

(a) The $H_{i}$ 's are homogeneous polynomials of degree $2 i$ of the form

$$
H_{i}=h_{i}\left(x_{1}^{2}+\xi_{1}^{2}, \ldots, x_{n}^{2}+\xi_{n}^{2}\right)
$$

( $h_{i}$ homogeneous of degree $i$ ), with

$$
H_{1}=\sum_{i=1}^{n} u_{i}\left(x_{i}^{2}+\xi_{i}^{2}\right) .
$$

(b) $R_{N}$ is homogeneous of degree $2 N$ and of the form:

$$
R_{N}=V_{N}+R_{N}^{\sharp},
$$

where $V_{N}$ consists of the terms homogeneous of degree $2 N$ in the Taylor series of $V\left(x_{1}^{2}, \ldots x_{n}^{2}\right)$ at $x=0$, and $R_{N}^{\sharp}$ is an artifact of the previous inductive steps.

(c) $R_{N}^{\prime}$ vanishes to order $2 N+2$ at the origin and is of the form

$$
R_{N}^{\prime}=V-\sum_{k=2}^{N} V_{k}+S_{N}
$$

where $S_{N}$ is another artifact of the inductive process. In addition, $R_{N}^{\prime}$ is even.

We will also show that this induction argument is such that one can read off from the $H_{i}$ 's the first $N$ terms in the Taylor expansion of $V\left(s_{1}, \ldots s_{n}\right)$ at $s=0$.

For $N=1$ in (2.5) these assertions are true with $\kappa$ the identity, $R_{2}=V_{2}$ consists of the quartic terms in the Taylor series of $V$, and $R_{2}^{\prime}=V-V_{2}$ (in particular $\left.R_{2}^{\sharp}=0=S_{2}\right)$.

Let us suppose that these assertions are true for $N-1(N \geq 2)$, so that

$$
\kappa^{*} H=\sum_{i=1}^{N-1} H_{i}+\tilde{R}_{N}
$$


with $\tilde{R}_{N}=R_{N}+R_{N}^{\prime}$, as above. We look for a homogeneous polynomial of degree $2 N, G_{N}$, such that

$$
\left\{H_{1}, G_{N}\right\}=R_{N}-H_{N}\left(x_{1}^{2}+\xi_{1}^{2}, \ldots, x_{n}^{2}+\xi_{n}^{2}\right)
$$

where $H_{N}\left(s_{1}, \ldots, s_{n}\right)$ is a homogeneous polynomial of degree $N$ in $s$. Introducing complex coordinates, $z_{i}=x_{i}+\sqrt{-1} \xi_{i}$, the Hamiltonian vector field

$$
\mathcal{V}=\sum_{i} \frac{\partial H_{1}}{\partial \xi_{i}} \frac{\partial}{\partial x_{i}}-\frac{\partial H_{1}}{\partial x_{i}} \frac{\partial}{\partial \xi_{i}}
$$

becomes the vector field

$$
\frac{2}{\sqrt{-1}} \sum_{i} u_{i}\left(z_{i} \frac{\partial}{\partial z_{i}}-\bar{z}_{i} \frac{\partial}{\partial \bar{z}_{i}}\right)
$$

in these coordinates, and

$$
x_{i}^{2}+\xi_{i}^{2}=z_{i} \bar{z}_{i}=\left|z_{i}\right|^{2} .
$$

Thus for $|\alpha|+|\beta|=2 N$,

$$
L_{\mathcal{V}}\left(z^{\alpha} \bar{z}^{\beta}\right)=\frac{2}{\sqrt{-1}}\left(\sum_{i} u_{i}\left(\alpha_{i}-\beta_{i}\right)\right) z^{\alpha} \bar{z}^{\beta} .
$$

Suppose $R_{N}=\sum_{|\alpha|+|\beta|=2 N} c_{\alpha, \beta} z^{\alpha} \bar{z}^{\beta}$. Letting

$$
G=\frac{\sqrt{-1}}{2} \sum_{\alpha \neq \beta} \frac{c_{\alpha, \beta}}{\langle u, \alpha-\beta\rangle} z^{\alpha} \bar{z}^{\beta}
$$

(where we are using the hypothesis of the linear independence of the $u_{i}$ over $\mathbb{Q}$ ), we get from $(2.10)$

where

$$
\left\{H_{1}, G_{N}\right\}=L_{\mathcal{V}} G=R_{N}-H_{N},
$$

$$
H_{N}=\sum_{\alpha=\beta,|\alpha|=N} c_{\alpha, \beta} z^{\alpha} \bar{z}^{\beta}
$$

These arguments prove:

Lemma 2.1. Given $R_{N}$ as above, there is a unique homogeneous polynomial, $G_{N}$, linear combination of monomials $z^{\alpha} \bar{z}^{\beta}$ with $|\alpha|+|\beta|=2 N$ and $\alpha \neq \beta$, such that (2.7) holds and where $H_{N}$ consists of the "diagonal" monomials of $R_{N}$.

For future reference we make a little more explicit the form of $G_{N}$. Note that

$$
x_{i}^{2 k_{i}}=\left(\frac{z_{i}+\bar{z}_{i}}{2}\right)^{2 k_{i}}=\left(\frac{1}{2}\right)^{2 k_{i}}\left(\begin{array}{c}
2 k_{i} \\
k_{i}
\end{array}\right)\left|z_{i}\right|^{2}+F_{k_{i}}+\overline{F_{k_{i}}},
$$

where

$$
F_{k_{i}}=\left(\frac{1}{2}\right)^{2 k_{i}} \sum_{0 \leq r<k_{i}}\left(\begin{array}{c}
2 k_{i} \\
r
\end{array}\right) z_{i}^{2 k_{i}-r} \bar{z}_{i}^{r} .
$$

This shows that

$$
x_{1}^{2 k_{1}} \cdots x_{n}^{2 k_{n}}=\left(\begin{array}{c}
2 k_{1} \\
k_{1}
\end{array}\right) \cdots\left(\begin{array}{c}
2 k_{n} \\
k_{n}
\end{array}\right)\left|z_{1}\right|^{2 k_{1}} \cdots\left|z_{n}\right|^{2 k_{n}}+\cdots
$$


where the dots are a linear combination of monomials of the form $z^{\alpha} \bar{z}^{\beta}$ with $\alpha \neq \beta$. Hence by (2.9) and (2.10) there exists a homogeneous polynomial, $G_{k}=G_{k_{1}, \ldots, k_{n}}$ of degree $2 N$ such that

$$
x_{1}^{2 k_{1}} \cdots x_{n}^{2 k_{n}}=\left(\begin{array}{c}
2 k_{1} \\
k_{1}
\end{array}\right) \cdots\left(\begin{array}{c}
2 k_{n} \\
k_{n}
\end{array}\right)\left|z_{1}\right|^{2 k_{1}} \cdots\left|z_{n}\right|^{2 k_{n}}+\left\{H_{1}, G_{k}\right\} .
$$

As we'll see below, this implies that, in solving (2.7), we can keep track of the Taylor coefficients of $V_{N}$ as terms in $H_{N}$ which are not artifacts of the previous steps in our induction.

Let $\mathcal{W}$ be the Hamiltonian vector field

$$
\mathcal{W}=\sum_{i} \frac{\partial G}{\partial \xi_{i}} \frac{\partial}{\partial x_{i}}-\frac{\partial G}{\partial x_{i}} \frac{\partial}{\partial \xi_{i}} .
$$

We will use the fact that, for any homogeneous function $F$, the Taylor series of the pull-back $(\exp \mathcal{W})^{*}(F)$ at the origin is given by the expansion

$$
(\exp \mathcal{W})^{*}(F) \sim \sum_{k=0}^{\infty}\left(\operatorname{ad}_{G}\right)^{k}(F),
$$

where $\operatorname{ad}_{G}(F)=\{G, F\}$. (Note that if $G$ is homogeneous of degree $l$ and $F$ is homogeneous of degree $m$, then $\{G, F\}$ is homogeneous of degree $m+l-2$.) With this in mind, by $(2.5)$

$$
\begin{gathered}
(\exp \mathcal{W})^{*} \kappa^{*} H=\kappa^{*} H+\left\{G, \kappa^{*} H\right\}+\cdots=\kappa^{*} H+\left\{G, H_{1}\right\}+\cdots \\
=\kappa^{*} H+H_{N}-R_{N}+\cdots=\sum_{i=1}^{N} H_{N}+\cdots
\end{gathered}
$$

where the dots represent terms that vanish to order $2 N+2$ at $(x, \xi)=(0,0)$. In fact, a calculation shows that for $N \geq 3$ the sum of the terms homogeneous of degree $2 N+2, R_{N}$, equals

$$
R_{N+1}=\left\{H_{2}, G\right\}+\left(R_{N}^{\prime}\right)_{2 N+2}
$$

where $\left(R_{N}^{\prime}\right)_{2 N+2}$ is the sum of the terms homogeneous of degree $2 N+2$ in the Taylor expansion of $R_{N}^{\prime}$. (For $N=2$ one has a couple of harmless additional terms, see below.) Thus if we replace $\kappa$ by $\kappa \exp (\mathcal{W})$, the inductive assumptions hold with $N-1$ replaced with $N$.

If we let $N$ tend to infinity in (2.5) we obtain the Birkhoff canonical form

$$
\sum_{i=1}^{\infty} H_{i}\left(x_{1}^{2}+\xi_{1}^{2}, \ldots, x_{n}^{2}+\xi_{n}^{2}\right)
$$

for the classical Hamiltonian (1.4).

2.3. Recovering the Taylor series of $V$. We now prove that if we are given the sequence of functions $\left\{H_{i}\right\}$ we can recover the Taylor series of $V$ at the origin.

We begin by noticing that $H_{1}$ consists precisely of the quadratic terms in the Taylor series. Moreover, the function $G_{2}$ in the first step of the inductive procedure above satisfies

$$
\left\{H_{1}, G_{2}\right\}=V_{2}-H_{2}
$$


(recall that $R_{2}=V_{2}$, i. e. $R_{2}^{\sharp}=0$ ). It is clear from (2.11) that the information in $V_{2}$ is encoded in $\mathrm{H}_{2}$; explicitly, if

$$
V_{2}=\sum_{k,|k|=2} c_{k} x^{2 k}
$$

then by $(2.11)$

$$
H_{2}=\sum_{k,|k|=2} c_{k}\left(\begin{array}{c}
2 k \\
k
\end{array}\right)|z|^{2 k}
$$

where we have let $x^{2 k}=x_{1}^{2 k_{1}} \cdots x_{n}^{2 k_{n}}$, etc. Therefore the quartic term, $V_{2}$, is determined by $\mathrm{H}_{2}$. This implies that $G_{2}$ is also determined (see Lemma 2.1).

If we now conjugate $H=H_{1}+V$ by the exponential of the Hamilton vector field of $G_{2}$, we obtain:

$$
\begin{gathered}
\kappa_{1}^{*} H=H_{1}+R_{2}+R_{2}^{\prime}+\left\{G_{2}, H_{1}\right\}+\left\{G_{2}, R_{2}\right\}+\left\{G_{2},\left\{G_{2}, H_{1}\right\}\right\}+O(8)= \\
=H_{1}+H_{2}+V_{3}+\left\{G_{2}, R_{2}\right\}+\left\{G_{2},\left\{G_{2}, H_{1}\right\}\right\}+O(8),
\end{gathered}
$$

where $O(8)$ stands for a function that vanishes to order eight at the origin. (We have: $\left.R_{2}^{\prime}=V-V_{2}=V_{3}+O(8).\right)$ Therefore

$$
R_{3}=V_{3}+\left\{G_{2}, R_{2}\right\}+\left\{G_{2},\left\{G_{2}, H_{1}\right\}\right\},
$$

the last two terms constituting $R_{3}^{\sharp}$, the first artifact of the inductive process. Notice, however, that $R_{3}^{\sharp}$ is known to us since $G_{2}$ was determined in the previous step.

The next inductive step involves the equation

$$
\left\{H_{1}, G_{3}\right\}=V_{3}+R_{3}^{\sharp}-H_{3},
$$

where one should notice that $R_{3}^{\sharp}$, and therefore its "diagonal" monomials, are known. Since $H_{3}$ is also known, arguing exactly as before (appealing to (2.11)), we see that $V_{3}$ and $G_{3}$ are determined by $H_{1}, H_{2}$ and $H_{3}$. It is clear now that one can continue indefinitely in this fashion.

Q. E. D.

2.4. The existence of a quantum Birkhoff canonical form. The existence of a quantum Birkhoff canonical form for the Schrödinger operator, (1.1), can be proved by essentially the same methods. Namely, let $p_{0}\left(s_{1}, \ldots, s_{n}\right)$ be a $C^{\infty}$ function with the same Taylor series as the series, $\sum_{i} H_{i}(s)$. Then, as we have just seen, there exists a canonical transformation, $\kappa$, conjugating (1.4) to the Birkhoff canonical form, $p_{0}\left(x_{1}^{2}+\xi_{1}^{2}, \ldots, x_{n}^{2}+\xi_{n}^{2}\right)$, modulo an error term which vanishes to infinite order at $x=\xi=0$. let $\mathcal{U}$ be a Fourier integral operator quantizing $\kappa$. Then

$$
\mathcal{U} P \mathcal{U}^{-1}=p_{0}\left(\hbar^{2} D_{1}^{2}+x_{1}^{2}, \ldots, \hbar^{2} D_{n}^{2}+x_{n}^{2}\right)+\hbar Q+R
$$

where the symbol of $R$ vanishes to infinite order at $x=\xi=0$. We will prove by induction that there exists an F. I. O., $\mathcal{U}_{N}$, such that microlocally near $x=\xi=0$

$$
\mathcal{U}_{N} P \mathcal{U}_{N}^{-1}=p_{0}\left(\hbar^{2} D_{1}^{2}+x_{1}^{2}, \ldots, \hbar^{2} D_{n}^{2}+x_{n}^{2}\right)+\hbar^{N} Q+R
$$

and the symbol of $R$ vanishes to infinite order at $x=\xi=0$. Assuming this assertion is true for $N-1$ let us prove it for $N$. Let the symbol of $Q$ be of the form $q=$ $q_{0}(x, \xi)+\hbar q^{\prime}(x, \xi, \hbar)$. We claim 
Lemma 2.2. There exist $C^{\infty}$ functions, $a(x, \xi)$ and $p_{N}\left(s_{1}, \ldots, s_{n}\right)$, such that

$$
\left\{p_{0}, a\right\}=q_{0}-p_{N}\left(x_{1}^{2}+\xi_{1}^{2}, \ldots, x_{n}^{2}+\xi_{n}^{2}\right)+r
$$

where $r$ vanishes to infinite order at $x=\xi=0$.

Proof. By (2.3), $p_{0}=H_{1}+p_{0}^{\prime}$ where $p_{0}^{\prime}(s)=O\left(s^{2}\right)$. Writing (2.15) in the form

$$
\left\{H_{1}, a\right\}=q_{0}-p_{N}-\left\{p_{0}^{\prime}, a\right\}+r
$$

it is clear that the homogeneous terms of degree $k$ in the Taylor series of $a$ and $p_{N}$ can be determined from the previous terms by $(2.7)$.

Now let $A=a^{W}(x, \hbar D)$ be the Weyl quantization of $a$, and let $V=$ $\exp \left(-\sqrt{-1} \hbar^{N} A\right)$. Then

$$
V\left(\mathcal{U}_{N-1} P \mathcal{U}_{N-1}^{-1}\right) V^{-1}=\mathcal{U}_{N-1} P \mathcal{U}_{N-1}^{-1}+\sqrt{-1} \hbar^{N}\left[\mathcal{U}_{N-1} P \mathcal{U}_{N-1}^{-1}, A\right]+\hbar^{N+1} Q^{\prime}+R^{\prime}
$$

where the symbol of $R^{\prime}$ vanishes to infinite order at $x=\xi=0$. In view of (2.14) and (2.15) the right hand side of this identity can be rewritten in the form

$$
\sum_{j=1}^{N} \hbar^{j} p_{j}\left(\hbar^{2} D_{1}^{2}+x_{1}^{2}, \ldots, \hbar^{2} D_{n}^{2}+x_{n}^{2}\right)+\hbar^{N+1} Q^{\prime \prime}+R^{\prime \prime}
$$

where the symbol of $R^{\prime \prime}$ vanishes to infinite order at $x=\xi=0$. Thus with $\mathcal{U}_{N-1}$ replaced by $V \mathcal{U}_{N-1}$ the identity (2.14) is valid with $N-1$ replaced by $N$, and letting $N$ tend to infinity one obtains the Birkhoff canonical form for $P$ described at the beginning of this section.

\section{An extension to the Riemannian case}

Let $M$ be an $n$-dimensional Riemannian manifold, $\Delta$ its Laplace-Beltrami operator, and $V: M \rightarrow \mathbb{R}$ a smooth function. We consider the Schrödigner operator $P=$ $\hbar^{2} \Delta+V$ on $L^{2}(M)$. We assume for simplicity that the spectrum of $P$ is discrete. In this section we describe an extension of Theorem 1.1 to $P$.

We will assume that $V$ has a unique global minimum, $m \in M$, and that it is nondegenerate. Then the Hessian of $V$ at $m$ is a well-defined positive-definite quadratic form on $T_{m} M$. Using the metric, we can speak of the eigenvalues, $u_{1}, \ldots, u_{n}$, of the Hessian. We assume that these eigenvalues are linearly independent over $\mathbb{Q}$. In particular all eigenvalues are distinct, and therefore $T_{m} M$ splits naturally as an orthogonal direct sum of lines (spanned by eigenvectors of the Hessian). Denote by

$G \cong \mathbb{Z}^{2^{n}}$ the group of linear transformations of $T_{m} M$, generated by the reflections $v_{i} \mapsto-v_{i}$, where $v_{1}, \ldots, v_{n}$ is a basis of eigenvectors of the Hessian.

Theorem 3.1. Assume that there exists a neighborhood, $\mathcal{U}$, of $m$ such that:

(1) $G$ acts on $\mathcal{U}$ by isometries.

(2) $m$ is a fixed point of the action, and the infinitesimal action on $T_{m}$ agrees with the original linear action of $G$.

(3) $\left.V\right|_{\mathcal{U}}$ is invariant under $G$.

Assume furthermore that $V(m)=0$. Then, assuming that we know the Riemannian metric on $M$, the eigenvalues (1.2) determine the infinite jet of $V$ at $m$. 
The proof is a straightforward generalization of the one given above in the Euclidean case. We will say a few words on how to show that the Birkhoff normal form of the classical Hamiltonian,

$$
H(x, \xi)=\|\xi\|^{2}+V(x)
$$

(where $(x, \xi) \in T^{*} M$ ), determines the Taylor series of $V$ at $m$.

Introduce geodesic normal coordinates centered at $m, x=\left(x_{1}, \ldots, x_{n}\right)$, such that the corresponding frame at $m$ consists of eigenvectors of the Hessian. In these coordinates the classical Hamiltonian takes the form

$$
H(x, \xi)=\sum_{i, j=1}^{n} g^{i j}(x) \xi_{i} \xi_{j}+V(x)
$$

where:

(1) $g^{i j}(x)=\delta_{i j}+O\left(|x|^{2}\right)$,

(2) $g^{i j}\left( \pm x_{1}, \ldots, \pm x_{n}\right)=g^{i j}\left(x_{1}, \ldots, x_{n}\right)$,

(3) $V\left( \pm x_{1}, \ldots, \pm x_{n}\right)=V\left(x_{1}, \ldots, x_{n}\right)$.

(The reason for $(2)$ and $(3)$ is that in the chosen coordinates $G$ acts by: $\left(x_{1}, \ldots, x_{n}\right) \mapsto$ $\left.\left( \pm x_{1}, \ldots, \pm x_{n}\right).\right)$ Proceeding as in $\S 3$, we can rewrite the Hamiltonian in the form

$$
H(x, \xi)=\sum_{i} u_{i}\left(x_{i}^{2}+\xi_{i}^{2}\right)+\sum_{i, j=1}^{n} h^{i j}\left(x_{1}^{2}, \ldots, x_{n}^{2}\right) \xi_{i} \xi_{j}+V\left(x_{1}^{2}, \ldots, x_{n}^{2}\right)
$$

where $V\left(s_{1}, \ldots, s_{n}\right)=O\left(s^{2}\right)$ and $h^{i j}\left(s_{1}, \ldots, s_{n}\right)=O(s)$.

The construction of the Birkhoff normal form $\sum_{j} H_{j}$ proceeds as before, except that the polynomials $R_{N}$ are now of the form

$$
R_{N}=\sum_{i, j=1}^{n} h_{N-1}^{i j}\left(x_{1}^{2}, \ldots, x_{n}^{2}\right) \xi_{i} \xi_{j}+V_{N}\left(x_{1}^{2}, \ldots, x_{n}^{2}\right)+R_{N}^{\sharp}
$$

where $h_{N}^{i j}\left(s_{1}, \ldots, s_{n}\right)$ consists of the terms homogeneous of degree $N$ in the Taylor series of $h^{i j}\left(s_{1}, \ldots, x_{n}\right)$. $H_{N}$ consists of the "diagonal" monomials in $R_{N}$, as before (that is, monomials of the form $c_{\alpha} z^{\alpha} \bar{z}^{\alpha}$ in complex coordinates $z_{j}=x_{j}+\sqrt{-1} \xi_{j}$ ).

We need to show that the sequence $\left\{H_{j}\right\}$ determines the Taylor series of $V$. This is possible because we are assuming that we know the metric, and therefore we know the sum $\sum_{i, j=1}^{n} h_{N-1}^{i j}\left(x_{1}^{2}, \ldots, x_{n}^{2}\right) \xi_{i} \xi_{j}$ appearing in $R_{N}$.

For example, $H_{2}$ consists of the diagonal terms in

$$
R_{2}=-\frac{1}{3} \sum_{i, j, k, l} R_{i k j l}(0) x_{k} x_{l} \xi_{i} \xi_{j}+V_{2}\left(x_{1}^{2}, \ldots, x_{n}^{2}\right) .
$$

If we subtract from $H_{2}$ the (known) diagonal terms in $-\frac{1}{3} \sum_{i, j, k, l} R_{i k j l}(0) x_{k} x_{l} \xi_{i} \xi_{j}$, we obtain the diagonal terms in $V_{2}\left(x_{1}^{2}, \ldots, x_{n}^{2}\right)$, which, as we have seen, determine the quartic terms in the Taylor expansion of $V$. The higher-degree cases are no different. 


\section{References}

[1] L. D. Landau and E. M. Lifshitz, Mechanics, Oxford, New York: Pergamon Press (1976).

[2] M. Dimassi and J. Sjöstrand, Spectral Asymptotics in the Semi-Classical Limit. Cambridge University Press (1999).

[3] V. Guillemin, T. Paul, A. Uribe, "Bottom of the well" semi-classical trace invariants. Preprint: math.SP/0608617.

[4] J. Sjöstrand, Semi-excited states in non-degenerate potential wells. Asymp. Anal. 6 No. 1 (1992), $29-43$.

Department of Mathematics, Massachusetts institute of Technology, Cambridge, MA 02139

E-mail address: vwg@math.mit.edu

Mathematics Department, University of Michigan, Ann Arbor, Mi 48109

E-mail address: uribe@umich.edu 